\title{
Development, validation and application of a generator for distortion product otoacoustic emissions
}

\author{
Mirjana Adnadjevic ${ }^{a, *}$, Annelies Bockstael ${ }^{a}$, Vincent Nadon ${ }^{\mathrm{a}}$, Pieter \\ Thomas $^{\mathrm{a}}$, Dick Botteldooren ${ }^{\mathrm{a}}$ \\ ${ }^{a}$ Department of Information Technology, Ghent University, St. Pietersnieuwstraat 41, \\ B-9000 Ghent, Belgium
}

\begin{abstract}
The application area of distortion product otoacoustic emissions (DPOAEs) is expanding beyond clinical routines, towards new measurement environments, protocols, wearable hardware and more robust algorithms. To enable systematic assessment of the performance of commercial and prototype OAE equipment under various measurement conditions without a need for recruitment of the test subjects, a DPOAE generator that produces a reliable and stable response is proposed. This article suggests simple design for such generator that is easy to reproduce by third parties. This design uses an approximate model for middle and inner ear dynamics focusing on typical operational conditions. The DPOAE generator is embedded in a head and torso simulator enabling testing for various probe fitting and also residual ear canal volume effects. Measurement of the DPOAE generator response with clinical equipment shows that the response obtained falls within the range of human normative data. It is also shown that the generator is applicable out-
\end{abstract}

\footnotetext{
*Corresponding author

Email address: madnadje@intec.ugent.be (Mirjana Adnadjevic)
} 
side the clinical routines and is able to highlight differences in measurements obtained with different clinical OAE measurement devices.

Keywords: otoacoustic emission, hearing screening, inner ear model, signal processing

PACS: 43.64.Jb, 43.64.Yp

\section{Introduction}

Otoacoustic emissions (OAEs) are low-level signals, primarily originating from outer hair cell activity located in the cochlea inside the inner ear. These OAE signals propagate back from the inner ear to the external ear canal where they can be observed using distortion-free recording and refined signal processing. Evoked OAEs (EOAEs) are OAEs in response to an external acoustical stimulus. Spontaneous OAEs (SOAE), on the other hand, occur without stimulus and are usually more rare in the human population. Within the EOAEs, the two most commonly used types are transient evoked OAEs (TEOAEs), evoked by click stimuli and distortion product (DPOAEs), evoked with pure tone stimuli $[1,2]$.

Both TEOAEs and DPOAEs are frequently used to monitor the status of the cochlea and detect potential outer hair cell damage. For example, EOAEs are now frequently used for diagnostic purposes and newborn screening $[3,4]$. For this and other clinical applications, protocols are developed to assure measurement reproducibility [5]. Also, technical standards for the electronic components in OAE equipment are available [6]. However, the field of application of OAE measurements is currently expanding. As an example, OAE measurements are also suggested as a tool for early detection of occupational 
and/or recreation noise-induced hearing damage, or for indirect monitoring of real exposure under hearing protector $[7,8]$. New devices are being developed to withstand the difficult measurement conditions that might occur outside the clinical environment. Manufacturers use different techniques to detect the OAEs and noise levels as the technology evolves. Developing new devices and test protocols requires extensive testing and validation.

At the moment, validation of OAE devices and their parameters is mostly done by repeated measurements with human test subjects. Due to intersubject and individual temporal variability of OAE responses, the test groups need to be sufficiently large and many repetitions are required. This imposes practical constraints on the number of measurement conditions that can systematically be compared. For this purpose, an OAE generator that produces realistic but time-invariant EOAE responses, would be useful. This generator has to be generic with respect to the various designs of the OAE equipment. All OAE devices, therefore, can operate on the generator as they would on human subjects.

The proposed design was intended for simulating DPOAE responses, since DPOAEs are well established in clinical practice, but are also more commonly used for in-field DPOAE measurements outside clinical routines [8]. The proposed DPOAE generator was developed starting from a standard headand-torso simulator (HATS). Such HATS based DPOAE simulator enables a human-like measurements setup including realistic probe placement in the outer ear canal. Also, it accounts for the influence of the head and torso on the sound propagation in elevated background noise levels. DPOAEs are generated in real time in response to stimuli sent by the OAE equipment. 
Within this hardware setup, various models could be implemented in signal processors for the fundamental DPOAE generation mechanisms - such as advanced models by Liu et al. [9], Neely et al. [10], Rapson et al. [11] and How et al. [12]. However, since the focus is on designing a generic tool, useful for the development and testing of OAE devices and protocols, a less complex DPOAE generator algorithm is proposed in this paper. The design is easy to reproduce by the third parties and it has low computational requirements.

The simulator produces $2 f_{1}-f_{2}$ DPOAE frequency component in responses to the primary frequencies $f_{1}$ and $f_{2}$. DPOAE responses are seen at multiple distortion products of $f_{1}$ and $f_{2}$, but numerous studies and clinical practice have focused on $2 f_{1}-f_{2}$ DPOAEs. Especially in humans, it is the strongest distortion component for a wide range of stimulus parameters [14]. There are no further restrictions or hypothesis that would limit the operational range of the proposed generator. However, to reduce the complexity of the generator model and to focus on hardware design, the following additional assumptions are made. The ratio $f_{1} / f_{2}$ is assumed to be around 1.22. In practice, this frequency ratio is the most adopted [13], as it assures that DPOAE amplitude at each frequency is still within $3 \mathrm{~dB}$ of its optimal value $[5,14]$. Furthermore, it is assumed that the proposed generator is intended to replicate DPOAE signals which are result of active cochlear processes observed for moderate stimuli levels ( $\leq 70 \mathrm{~dB}(\mathrm{SPL}))$ [5] in subjects with normal hearing. Moderate stimuli levels are used in conventional clinical protocols for hearing screening, as low level stimuli enhance the sensitivity of DPOAE responses to cochlear dysfunction [5, 13], while high level stimuli can lead to false-high DPOAE responses due to the system distortion [15]. 
The generator is further simplified by assuming that the level of the tone produced at frequency $f_{1}\left(L_{1}\right)$ is significantly higher (e.g. $10 \mathrm{~dB}$ ) than the level produced at frequency $f_{2}\left(L_{2}\right)$. This level difference is known to lead to the optimal levels of the DPOAE responses [16].

The basic principles of the proposed DPOAE generator have already been introduced by authors in [17]. The design is explained in detail in Section 2. Sections 3 and 4 describe the methodology and results of an extensive validation of the DPOAE generator responses with normative data obtained with the human subjects in the quiet clinical conditions [18]. For this purpose, a newer type of OAE equipment is used compared to the one used in [17]. In addition, Sections 3 and 4 explain two examples of use of the DPOAE generator. As the first example, DPOAE responses are measured in elevated background noise conditions. The results are also compared with reported data on the human subjects [19]. Another example of the applicability compares the DPOAE responses measured by two different commercial OAE devices. Finally, the performance of the proposed DPOAE generator is analyzed and discussed in Section 5.

\section{Design of the DPOAE Generator}

\subsection{Hardware Components}

The proposed DPOAE generator is integrated in commercially available HATS with middle and outer ear simulator, in casu Brüel \& Kjær HATS type 4128C (Figure 1, Brüel \& Kjær, Denmark). The OAE measurement device under test (DUT) and its OAE probe generate primary tones and record the DPOAE responses. Stimuli sent by the OAE probe are picked 


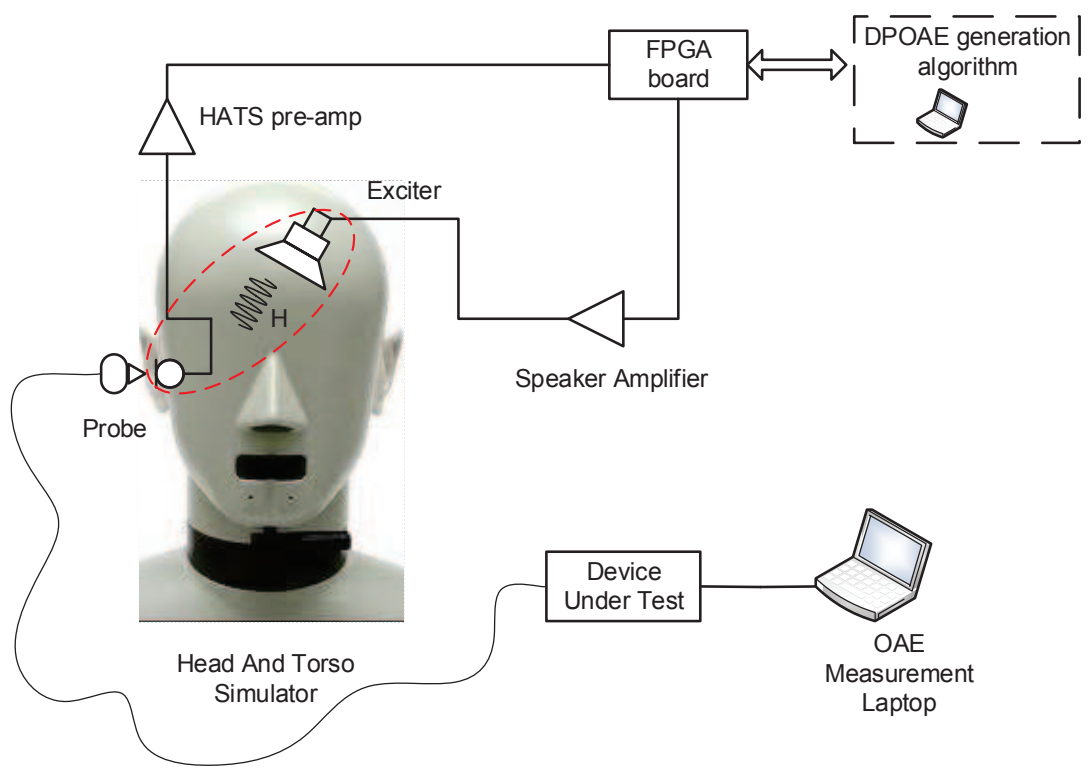

Figure 1: DPOAE generator - hardware components

up by the sensitive microphone of the HATS, passed through the HATS preamplifier and are further routed through the Field-Programmable Gate Array (FPGA) interface to a personal computer where the fundamental mechanisms of DPOAE responses are simulated (see subsection 2.2). Note that the FPGA is used solely for acquisition and playback and can be replaced by any high quality audio card. Subsequently, the DPOAE responses are evoked with a mid-frequency exciter, type VISATON EX 45 S (VISATON, Germany), attached to the ear simulator block inside the HATS (Figure 1).

In this configuration, the generated DPOAE responses are altered by the propagation from the exciter via the ear simulator block to the ear canal. The transfer function between the exciter and the HATS microphone in the 
ear canal ( $\mathrm{H}$ in Figure 1) depends on the hardware that is used and can be rather complex (see Figure 2). To compensate for it and to preserve the desired DPOAE levels in the ear canal, a digital filter is included in the DPOAE response generation path $\left(H^{-1}\right.$ in Figure 3). If another type of the HATS, middle ear simulator, exciter and/or probe placement is used, the compensation filter $\left(H^{-1}\right)$ is automatically adapted. Therefore, this compensation procedure allows for flexible adaptation to and independence of the choice of hardware fixture.

The compensation filter $\left(H^{-1}\right)$ is identified prior to the DPOAE measurement using an adaptive normalized least mean square (NLMS) technique. The inverse transfer function $\left(H^{-1}\right)$ is implemented as a Finite Impulse Response (FIR) filter of order $N=1024$ with a delay of 500 samples for a sampling frequency of $50 \mathrm{kHz}$. By compensating for the hardware-related transfer function in this way, the desired DPOAE signals are produced in the ear canal. The physical transfer function ( $\mathrm{H}$ in Figure 1) and its electronically identified inverse transfer function $\left(H^{-1}\right.$ in Figure 3$)$ are shown on the Figure 2 .

During the design process of the DPOAE generator, different exciter models and exciter positions have been tested in terms of output levels and frequency response. A miniature loudspeaker placed directly in the ear canal was also considered as a possibility. However, adding the loudspeaker in the ear canal would alter the impedance in the ear canal and hence the working conditions of the OAE probe in the generator compared to human subjects. 


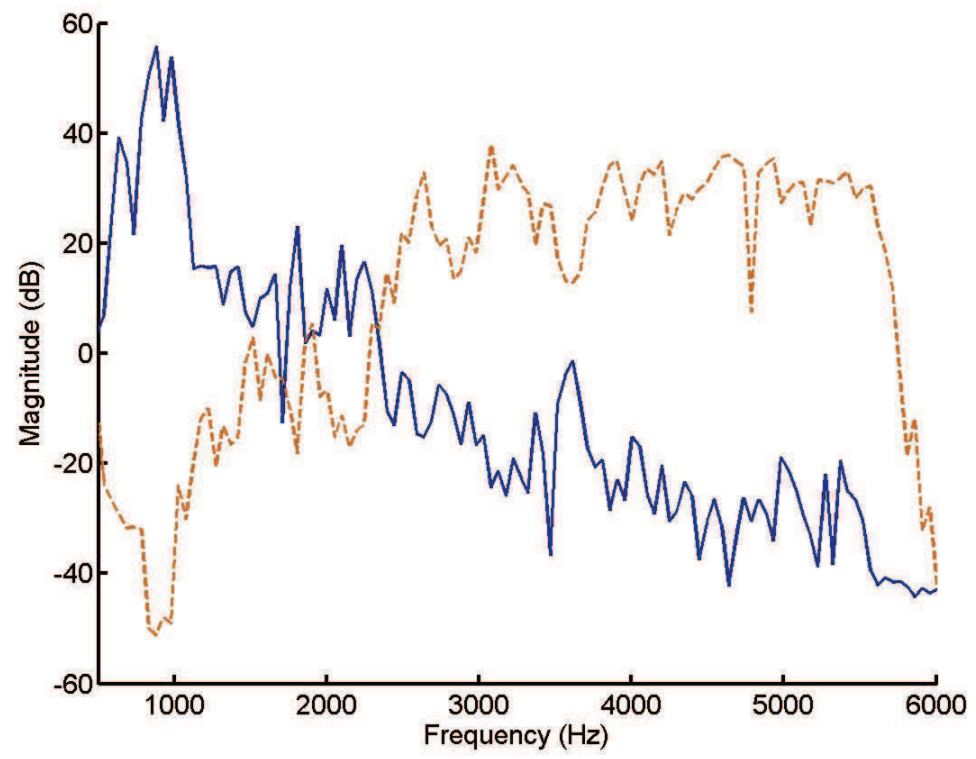

Figure 2: Transfer function from the exciter to the ear canal ( $\mathrm{H}$, full line) measured by the HATS microphone and the inverse transfer function $\left(H^{-1}\right.$, dashed line).

\subsection{DPOAE generation algorithm}

The block diagram of the DPOAE generation algorithm proposed in this paper is shown in Figure 3. Here, the middle ear filter (MEF) represents the influence of the middle ear on the DPOAE response. This influence has to be accounted for since the middle ear affects both the stimuli signals propagating from the ear canal to the inner ear via the middle ear, and the DPOAEs generated by the cochlea, propagating back via the middle ear to the ear canal. Therefore, the middle ear influence is addressed as a two port system with a forward and backward function [20]. In our model, these functions are represented by two FIR filters (MEF: M1, M2) of the same order $N=1024$. The filters approximate the average forward (M1) and 


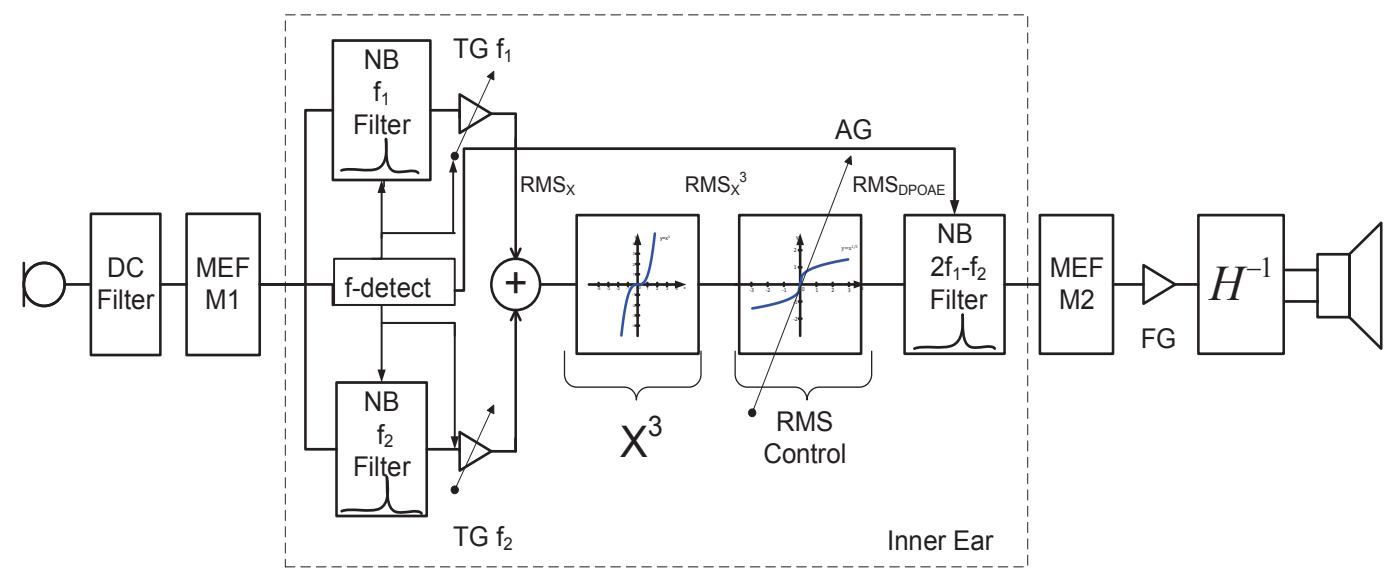

Figure 3: DPOAE generation algorithm

backward (M2) middle ear pressure gain, measured on thawed ears of human cadavers [20] and are shown in Figure 4.

The part of the block diagram in-between the middle ear filters models the inner ear. In the proposed design, the DPOAE response at frequency $2 f_{1}-f_{2}$ is generated using a third power function $\left(X^{3}\right)$. This function is the lowest integer power that produces the distortion product at the frequency $2 f_{1}-f_{2}$. In addition, it can easily be implemented in analogue electronics if one desires a more compact hardware design.

The third power function introduces a supralinear increase in DPOAE magnitudes with stimulus magnitudes. Hence, a compression factor is included in the algorithm, accounting for the compression mechanisms found in healthy human cochleas [15]. The compression factor is set at $0.3 \mathrm{~dB} / \mathrm{dB}$ according to the results reported by Dorn et al [15] for moderate stimuli levels. This means that a $1 \mathrm{~dB}$ increase in stimulus level leads to a $0.3 \mathrm{~dB}$ increase in DPOAE response level. This factor is kept constant over the range 


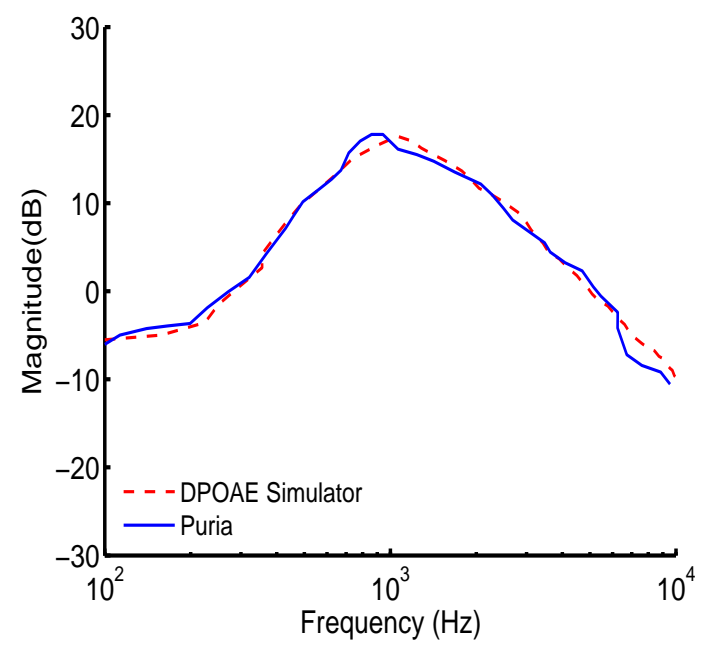

(a) MEF M1 filter

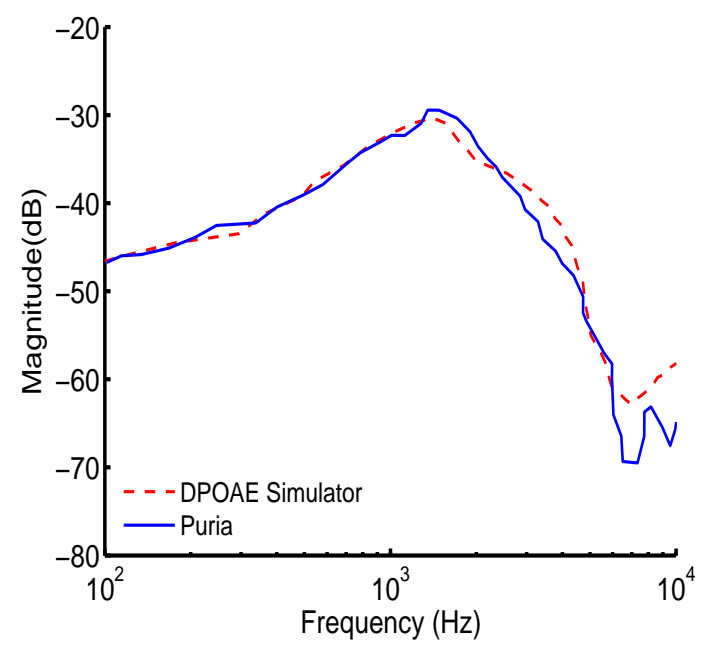

(b) MEF M2 filter

Figure 4: Middle ear forward (M1) (a) and backward (M2) (b) pressure gain used in the DPOAE simulator, based on the measurements by Puria [20]

of stimulus frequencies of interest. In the DPOAE generator, compression is achieved by monitoring continuously the ratio of the root mean square levels (RMS) of the signals before $\left(R M S_{x}\right)$ and after $\left(R M S_{x^{3}}\right)$ the third power and calculating the adjustable gain (AG) to approximate the desired compression factor. To illustrate how the RMS based gain control works, assume that the tones at the primary frequencies $\left(f_{1}, f_{2}\right)$ are presented at the ear drum at levels $L_{1}$ and $L_{2}=L_{1}-10 \mathrm{~dB}$. With this $10 \mathrm{~dB}$ level difference, the RMS level at input and output of the third power function is dominated by the $f_{1}$ primary component and:

$$
R M S_{x} \approx A, R M S_{x^{3}} \approx A^{3}=>\frac{R M S_{x^{3}}}{R M S_{x}} \approx A^{2},
$$

where $R M S_{x^{3}}$ and $R M S_{x}$ are the RMS values at the output and input of 
the third power function respectively and $A$ is the RMS amplitude of the $f_{1}$ frequency component $\left(A=2 \cdot 10^{-5} \cdot 10^{\frac{L_{1}}{20}}\right)$. Based on Eq. 1 and the compression factor requirement, the following adjustable gain $(A G)$ is proposed as:

$$
A G=\frac{R M S_{D P O A E}}{R M S_{x^{3}}} \approx \frac{A^{0.3}}{A^{3}}=\left[\frac{R M S_{x^{3}}}{R M S_{x}}\right]^{-1.35},
$$

where $R M S_{D P O A E}$ is the RMS value of the target DPOAE magnitude.

To mimic the cochlear tuning properties, narrow-band filters (NB) around automatically detected primary tones are introduced. They are designed as frequency following Infinite Impulse Response (IIR) Butterworth filters of order $N=3$, with a $Q$-factor of 50 . The central filter frequency is continuously adjusted through a frequency detection procedure. This filtering is a simplified representation of the tonotopic mapping within the cochlea, without replicating in detail the cochlear tuning curve properties. With the proposed $Q$-factor, the equivalent rectangular bandwidth (ERB), which is a measure for the tuning curve selectivity, is narrower compared to what was introduced in the model by Liu et al. [9] and measured by Gorga et al. [21]. Allowing for the higher $Q$-factor is reasonable, since Liu et al. also stated that further improvements of their model is needed in order to obtain sharper tuning properties. This is in agreement with findings measured on humans by Glasberg and Moore [22] and Shera et al. [23].

The relative amplitude between tonotopic curves is accounted for by introducing - frequency dependent - tonotopic gains, indicated in Figure 3 as $T G f_{1}$ and $T G f_{2}$. The gains are derived from the tonotopic map model, proposed by Liu et al. [9]. They approximate observations by a near-linear dependency between the logarithmic frequency and the gain level at the peak 
of the tuning curve. Therefore, frequency dependent tonotopic gains are calculated as follows (Eq. 3):

$$
T G_{\left(f_{i}\right)}(d B)=24 \log \left(f_{i}\right)-27 \quad \text { for } \quad i=1,2
$$

An additional narrow-band filter is applied around the distortion component. Although not directly related to the inner ear mechanics, suppressing the primary tones and other third power distortion products substantially improves the DPOAE signal-to-noise ratio. A final gain control (FG) is used to tune the overall DPOAE level to normative data [18].

\section{Methodology for validation and example applications}

\subsection{Validation of the DPOAE generator}

For validation of the device, response of the DPOAE generator has been measured with the ILO 292 USB II hardware and ILO v6 software (Otodynamics Ltd., United Kingdom). The OAE probe was calibrated before each measurement session with the 1cc calibration cavity, provided by the manufacturer. The optimal probe fit in the ear canal was ensured by performing the ILO system's check-fit procedure, using the click stimulus before each measurement.

The $2 f_{1}-f_{2}$ DPOAEs were measured by the ILO device by simultaneous presentation of two primary tones with a fixed primary tone level $\left(L_{1}=65 \mathrm{~dB}(\mathrm{SPL}), L_{2}=55 \mathrm{~dB}(\mathrm{SPL})\right)$ and frequency ratio $f_{2} / f_{1}=1.22$. The frequency of the second primary tone ranged from $841 \mathrm{~Hz}$ to $8000 \mathrm{~Hz}$. It was presented at eight frequency points per octave resulting in a 27 fre-

quency points DPOAE response. In the test sequence, stimuli excitation 


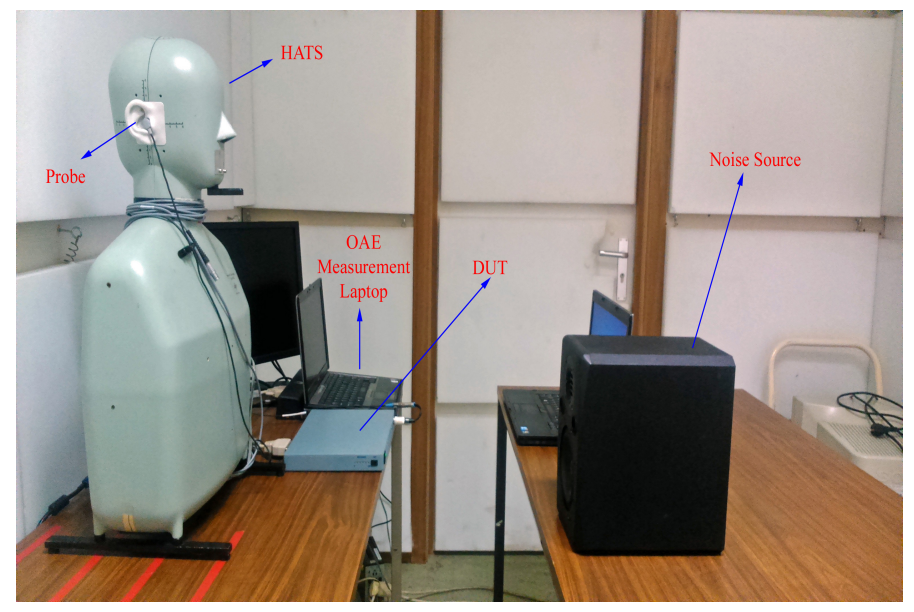

Figure 5: Measurement setup

and pause for switching between consecutive frequency combinations took in total 1.4 seconds. The measurement was stopped after the whole DPOAE frequency range was covered twice. A noise artifact rejection level of $8 \mathrm{mPa}$ was used. For the DPOAE signals to be included as valid, no a-priori signalto-noise ratio was set. However, DPOAE amplitudes corresponding to the detection floor of the system (in casu $-30.0 \mathrm{~dB}$ ) were rejected. All validation measurements were carried out in a quiet listening room (Figure 5), corresponding to the clinical conditions. They were repeated within one test day without refitting the OAE probe and across different test days with probe refit to assess the stability of the DPOAE generator and the proposed measurement procedure. Results are compared with the normative data acquired on humans, obtained with the same OAE equipment and user defined settings [18]. 


\subsection{Application of the DPOAE generator: measurement in elevated back-}

ground noise

The first application example assesses the possibility for performing DPOAE measurements with a clinical system in noisy measurement conditions. This has been tested by adding background noise in the listening room (noise source in Figure 5). During the tests, white noise fragments were presented through an Adam Audio S1X loudspeaker (Adam Audio, Germany) with built-in amplifier. The speaker, placed $78 \mathrm{~cm}$ from the HATS, was connected to a laptop with an U24XL sound card (ESI Audiotechnik GmbH, Gremany). Background noise levels were increased gradually from $50 \mathrm{~dB}(\mathrm{~A})$ up to the limit where the DPOAE measurement device could not preform accurate measurements anymore. DPOAEs were measured with the ILO 292 USB II hardware and ILO v6 software (Otodynamics Ltd., United Kingdom) connected to a laptop. The measurement settings were kept the same as for the quiet conditions, except that during the test sequence the frequency range is covered four times instead of two. This improved signal-to-noise ratio and allowed to compare results with [19] where also four repetitions were used.

Prior to the measurements, the background noise levels were measured with a Svantek sound analyzer (Svantek, Poland) at the HATS position without the HATS being present. $\mathrm{L}_{\text {Aeq }}$ levels were logged in $1 / 3$ rd octave bands with center frequencies from $20 \mathrm{~Hz}$ to $20000 \mathrm{~Hz}$ every second during the experiment. Table 1 gives an overview of the $\mathrm{L}_{\text {Aeq,1s }}$ of background noise fragments for which the ILO system was able to perform accurate DPOAE measurements. After this background noise level calibration procedure, the HATS was put back in place and DPOAE responses generated by the gener- 


\begin{tabular}{|c|c|}
\hline No. & Noise $\mathrm{dB}(\mathrm{A})$ \\
\hline 1 & 50.0 \\
2 & 53.0 \\
3 & 58.0 \\
\hline
\end{tabular}

Table 1: Overview of total white noise $\mathrm{L}_{\text {Aeq,1s }}$ background noise levels, recorded prior to measurements with the DPOAE generator.

ator were recorded.

\subsection{Application of the DPOAE generator: Comparison of clinical devices}

To illustrate how the DPOAE generator can be used to assess in detail the DPOAE values given by different OAE measurement devices for the same emissions, a second device was tested. For this comparison, an older ILO 292 DPEchoport system (Otodynamics Ltd., United Kingdom) was used. Again, the same ILO v6 software was used. In addition, all user-defined settings were kept the same. It is known however from the manufacturer information that the frequency range of this equipment is smaller, ranging from $1000 \mathrm{~Hz}$ to $6169 \mathrm{~Hz}$. Comparison measurements were carried out in quiet conditions.

\section{Results}

\subsection{Validation}

As explained in the Section 3.1, the DPOAE generator was tested with the DPOAE measurement device (ILO 292 USB II), widely used in the clinical practice. The proposed DPOAE generator is a valid alternative for repeated measurements on humans if: 1. the DPOAEs fall within the range of human 


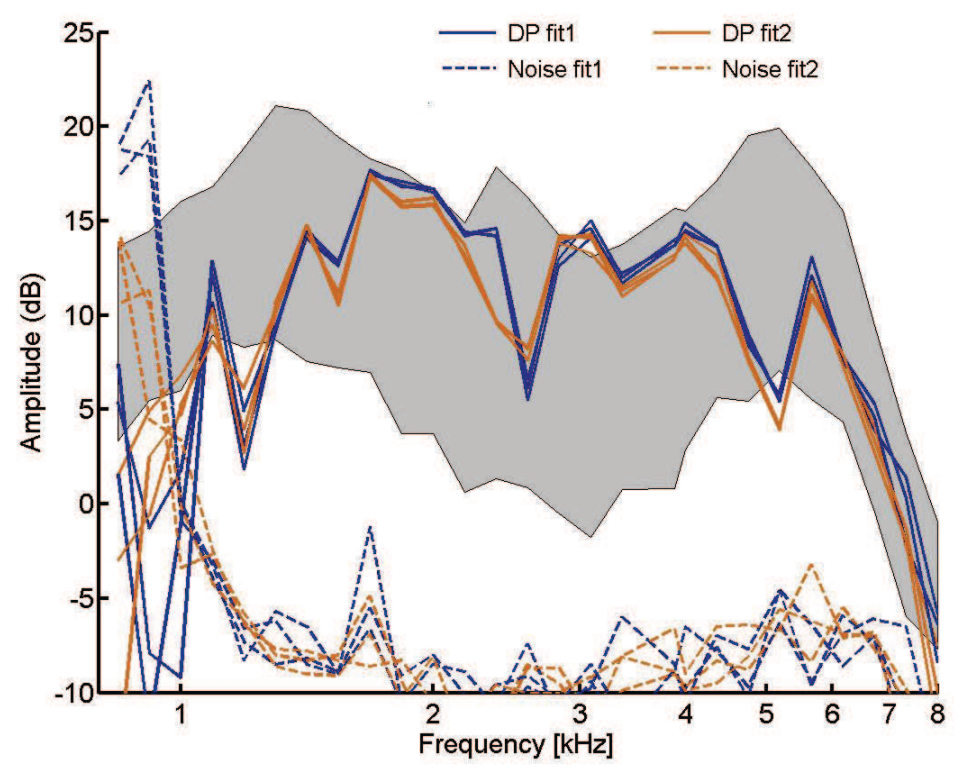

Figure 6: DPOAE signal and noise level measured by the same commercial OAE equipment for two different probe fits (DP fit1, DP fit2). In shaded grey area, normative DPOAE levels are shown, spanning the average DPOAE levels obtained for normal hearing subjects plus and minus one standard deviation [18].

DPOAEs and thus are representative; 2. the generator produces the DPOAEs in a reproducible way with or without refitting the OAE device under test. Figure 6 shows the amplitude of the DPOAE signals measured by the ILO device for the repeated trials with and without refit of the OAE probe.

For the $f_{2}$ frequencies above $1 \mathrm{kHz}$ the signal-to-noise ratio $(\mathrm{S} / \mathrm{N})$ clearly exceeds $3 \mathrm{~dB}$. In clinical terms, signals with this $\mathrm{S} / \mathrm{N}$ are interpreted as 'true' DPOAE responses and not artifacts [5, 24]. DPOAE amplitudes produced by the proposed generator fall within the range of normative data obtained on normal-hearing subjects between 18 and 30 years old [18]. Deviations 


\begin{tabular}{|c|c|c|c|c|c|c|c|}
\hline Frequency $(\mathrm{kHz})$ & 1 & 1.4 & 2 & 2.8 & 4 & 6 & 8 \\
\hline Variance $(\mathrm{dB})$ & 4.62 & 0.17 & 0.09 & 0.45 & 0.25 & 0.47 & 1.11 \\
\hline
\end{tabular}

Table 2: DPOAE variance without probe refit in clinical conditions.

observed for DPOAEs at $f_{2}$ frequencies below $1 \mathrm{kHz}$ will be discussed in detail in Section 5 .

Overall, repeatability of the measurements without probe refit is high as can be seen from the consecutively measured responses in Figure 6. Table 2 gives the variance of DPOAEs for 7 frequencies corresponding to the halfoctave frequency bands in the range $(1 \mathrm{kHz}-8 \mathrm{kHz})$. In the range roughly spanning from $1.4 \mathrm{kHz}$ to $6 \mathrm{kHz}$, the variance is below $0.5 \mathrm{~dB}$. In comparison to the findings on human subjects [25], the generator avoids intra-subject variability in this mid-frequency region. For frequencies around $8 \mathrm{kHz}$, the variance is increased to $1 \mathrm{~dB}$. This finding correspond with what was previously reported on human subjects [25]. The least stability and, therefore, the poorest reliability of DPOAE responses is, for both generator and the human subjects, seen around $1 \mathrm{kHz}$. The main cause is usually external lowfrequency environmental noise reaching the microphone of the probe through coupling transmission [25]. The poorer performance of the proposed DPOAE generator compared to the human case below $1 \mathrm{kHz}$ is elaborated in the discussion. Repeated measurements with probe refit also confirm the stability of the generated responses for the frequencies above $1 \mathrm{kHz}$. 


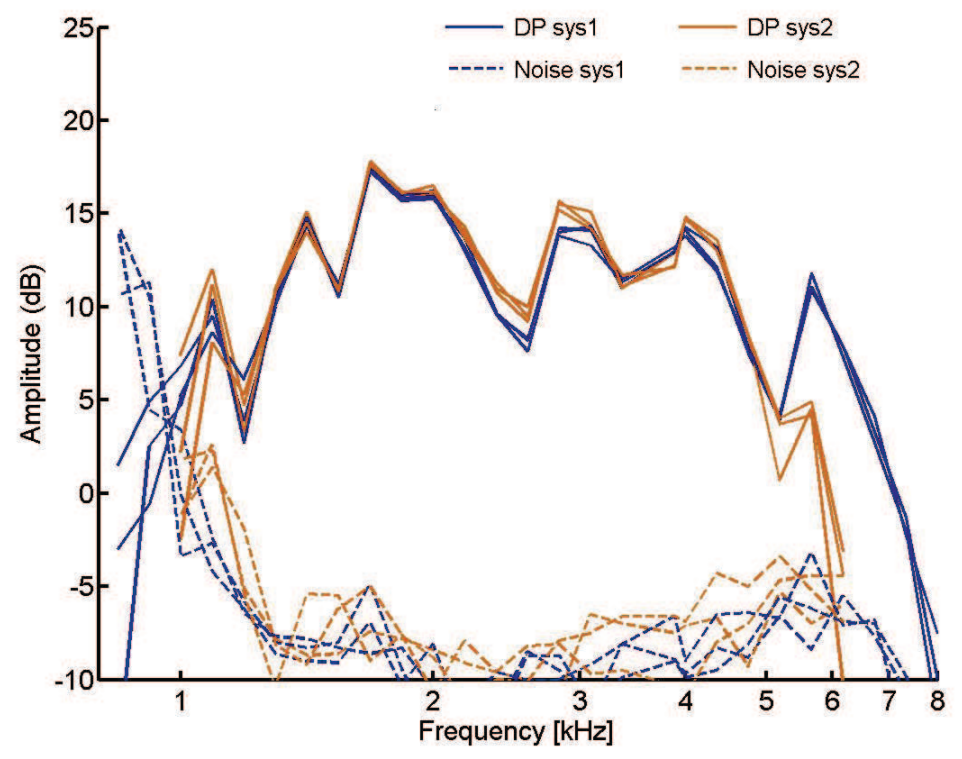

Figure 7: Repeated DPOAE simulator responses without probe refit measured in quiet test conditions (Silence) and in white noise levels of 50, 53 and $58 \mathrm{~dB}(\mathrm{~A})$.

\subsection{Application of the DPOAE generator: measurement in noise}

Figure 7 shows the results of DPOAE responses obtained with proposed generator in quiet, clinical conditions (Silence) and in white noise levels of 50, 53 and $58 \mathrm{~dB}(\mathrm{~A})$ (conditions 1-3 in Table 1). Such conditions were tested to study the effect of elevated background noise levels on DPOAE responses with proposed DPOAE generator.

DPOAE responses were measurable in white noise fragments up to $58 \mathrm{~dB}(\mathrm{~A})$. For higher levels, excessive noise present in the ear canal decreased the signal-to-noise ratio and the DPOAE measurement system under test failed to record the DPOAEs. Within this range of tolerable background noise levels (58 dB(A)), the DPOAE responses appeared not to be markedly influ- 


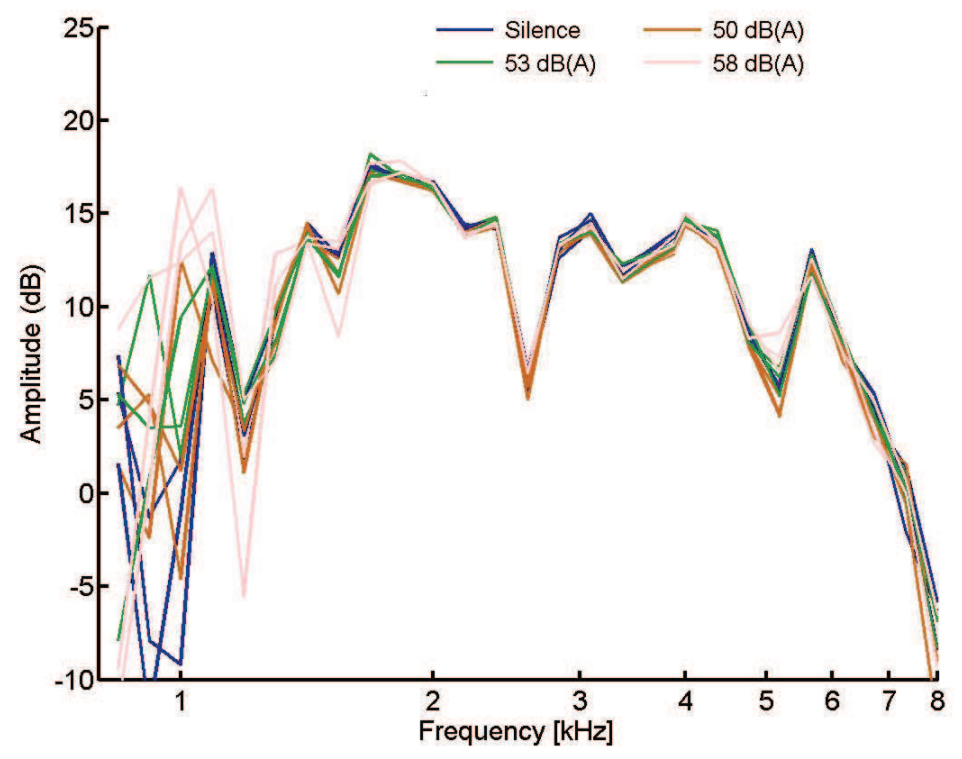

Figure 8: DPOAE signal and noise level produced without probe refit by ILO 292 USB II (DP sys1) and ILO 292 DPEchoport (DP sys2) commercial OAE devices.

enced by the noise conditions (Figure 7). Between the different background noise conditions, the DPOAE response variations are within $1 \mathrm{~dB}$ for the frequencies above $1 \mathrm{kHz}$. However, below $1 \mathrm{kHz}$ unstable generator DPOAE amplitudes are also present in higher background noise levels (see Section 5 for an extensive discussion).

\subsection{Application of the DPOAE generator: Comparison between clinical OAE devices}

In order to show that the DPOAE generator is indeed suitable for the comparison of different DPOAE processing algorithms, hardware and settings, DPOAE responses of the proposed generator were measured with two 
OAE measurement devices. Figure 8 shows the DPOAE responses obtained with ILO 292 USB II and ILO 292 DPEchoport OAE devices. In the frequency range spanning roughly from $1 \mathrm{kHz}$ to $4.5 \mathrm{kHz}$, DPOAEs measured by both systems are very close to each other. Differences are of the order of magnitude of the differences observed when refitting the probe. By contrast, the ILO 292 DPEchoport measures lower DPOAE amplitude for the three highest frequency points. The drop in DPOAE amplitude for frequencies above $4.5 \mathrm{kHz}$ could be related to the ILO 292 DPEchoport system working close to its frequency limits. Manufacturers of OAE equipment in general do not provide normative data nor specifications on algorithms used. Therefore, using an artificial OAE generator, such as the one presented in this paper, to compare equipment could allow clinicians to differentiate the variations in data due to the measurement equipment from those due to the physiology/pathology of the human ear under test.

\section{Discussion}

A DPOAE generator with a simple design that could be reproduced easily by any researcher or equipment developer interested in a HATS-featuring human-like DPOAEs was presented. The device was designed to allow fitting OAE equipment in exactly the same way as one would on a human subject. Therefore, it opens opportunities for designing probes, sound generation, signal acquisition and signal processing for clinical as well as harsh environments. The time independent character of generator, compared to humans, also allows exploring reproducibility of probe fit and comparing different measurement devices. 
A comparison with normative data in clinical conditions [18] showed that for frequencies above $1 \mathrm{kHz}\left(f_{2}\right)$, the DPOAE generator is capable of generating realistic responses that are stable in time, apart from expected test-retest variability due to differences in probe placement. Below $1 \mathrm{kHz}$, the noise level increases considerably and the amplitude of the DPOAE responses falls below the normative data. Higher variations are also seen across repeated tests.

These observations could be related to a - Helmholtz type - resonance of the occluded ear canal when a small leak exists. Such a resonance is also seen in the transfer function between the exciter and the HATS microphone shown in Figure 2. Authors in [26] reported the same abberation during carefully repeated tests with the same HATS type and measurement setup (occluded ear). The resonance is probably more pronounced than in humans due to the additional leak that might exist between the artificial ear and the ear canal and middle ear simulator. This clearly explains the higher noise levels at low frequencies both in the presence and absence of external background noise.

The low frequency resonance also explains the lower DPOAEs in two ways. Firstly, OAE measurement devices need a minimal $\mathrm{S} / \mathrm{N}$ ratio to be able to detect DPOAE signals. The higher noise level around $1 \mathrm{kHz}$ brings this $\mathrm{S} / \mathrm{N}$ ratio significantly down (see Figure 6 ). Secondly the compensation filter $H^{-1}$ might overcompensate for the resonance since it is based on measurement of the transfer function to the HATS microphone rather than the microphone of the OAE equipment. The latter is not available to the designer of the OAE generator. DPOAE responses below $1 \mathrm{kHz}$ could be boosted in the OAE generator by adapting the compensation filter $\left(H^{-1}\right)$. However, this would make the generators design tuned to one specific hard- 
ware and the procedure for compensating for exciter transfer function less general. Notwithstanding this minor flaw due to the HATS, the DPOAE generator was shown to be useful in two example situations.

In the first application example, proposed generator is used to examine the performance of the clinical OAE equipment under test when used in elevated background noise. The aim is to better understand whether and how the higher noise levels influence DPOAE measurements using clinical equipment and up to what background noise levels this equipment can be used. This is essential as the DPOAEs are becoming an objective, fast and reliable way to detect early signs of noise-induced changes in hearing sensitivity $[7,8]$ and a tool for direct monitoring of the efficiency of hearing protection $[7,8]$.

For the OAE measurement device under test, the DPOAE levels measured in elevated background noise levels remain the same up to (white) noise levels of $58 \mathrm{~dB}(\mathrm{~A})$ with a maximal change of $1 \mathrm{~dB}$. This indicates that the standard OAE equipment is able to suppress any influence of this noise on the DPOAE measurements. This conclusion is similar to the conclusion drawn from measurements obtained with a large group of human subjects [19], where variations of around $2 \mathrm{~dB}$ are reported for the same range of background noise levels. Also, the limit of $58 \mathrm{~dB}(\mathrm{~A})$ above which measurement of DPOAE fails is similar to the limit obtained from measurements with humans [19]. However, the failure to detect DPOAEs in noisy measurement conditions takes a slightly different form when using the generator compared to doing measurements on a cohort of persons. In the latter case, DPOAEs are occasionally detected in more elevated noise levels since some persons have higher DPOAE level by nature. The artificial generator 
therefore has to be regarded as representative for a single individual drawn from the population. The DPOAE generator can support the development of hardware and software solutions that allow extending the range of tolerable background noise levels [7]. This would enabling robust DPOAE acquisition in even harsher measurement environments.

The fact that DPOAE responses are low-level signals makes recordings largely dependent on the quality of the acquisition. The criteria for accurate acquisition and acceptable noise floor can vary among the devices depending on the manufacturer's recommendations [5]. Processing algorithms to distinguish DPOAE responses from artifacts also vary among the manufacturers [5].

As an example of applying the proposed DPOAE generator as a common reference, DPOAE responses have been compared for newer and older device type from the same manufacturer (Otodynamics Ltd.). For both OAE measurement devices, the same coupler-based calibration of the stimuli level was done first, following the built-in calibration procedure as suggested by the manufacturer's user guide. Differences in DPOAE level were seen at the highest frequencies. This example shows that improvements in OAE equipment can have a substantial influence on the final DPOAE amplitudes. This is also important outside routine clinical conditions, where constantly advancements are being made to enable DPOAE acquisition in suboptimal test conditions [7, 27, 28].

Apart from using an artificial DPOAE generator as a reference, alternative approaches could be used to assess and improve the comparability between DPOAE responses acquired with different systems. Monitoring of 
the primary tone stimulus levels might help to anticipate on equipmentrelated differences in DPOAE responses. Still, this will not cover the effect of improved processing algorithm on the response. Additionally, there is the IEC standard on electroacoustics for OAE instrumentation [6]. However, this standard is to-date not used by all manufacturers. It does not cover the processing used and it does not necessarily cover the whole range of the OAE measurement procedures and/or measurement conditions.

Another alternative to study the effect of methodological changes on the DPOAE responses could be through the separation of the OAE measurement device under test into smaller subsystems. The transfer function of every subsystem could then be measured and compared. However, this would be a tedious work, very sensitive to additional non-linearities such as measurement probe and ear canal non-linearities. Moreover, such a check could not be done by an end-user, as most algorithms and parameters used in commercially available OAE devices are not generally accessible.

Considering these difficulties, the stable and time-invariant, hence reproducible, DPOAE responses generated by the proposed generator offer an added value. Although the deviations from normative human data for the lower frequencies leave room for improvement, good agreement is seen between the DPOAE generator and the human data for the largest part of the frequency range. As most acoustic test fixtures, the proposed DPOAE generator can never fully replace validation and testing with human subjects. However, it is considered as an additional and time-saving tool to assess (new) DPOAE test protocols, develop and test (new) measurements devices, and design more robust algorithms. 


\section{Conclusions}

A reference tool for DPOAE measurement devices, named here DPOAE generator, was successfully designed. This tool has limited latency and thus allows for real-time generation of DPOAEs. It generates human like responses for primary tones with a frequency ratio of approximately 1.22 and stimulus amplitudes around $65 \mathrm{~dB}$. Because of careful balancing complexity and accuracy in its design, it remains easy to implement by any researcher or developer eager to test their DPOAE equipment on a reference device.

Comparing the response of the generator with normative data for human subjects acquired in quiet clinical conditions has shown that the proposed DPOAE generator is a reliable tool. Also, stability of the responses obtained in elevated background noise conditions extend the application area outside the clinical practice. Moreover, comparison of DPOAE responses measured by two different commercial OAE measurement devices has shown that the DPOAE generator is useful to asses differences in the DPOAE measurement devices' processing.

The DPOAE generator is therefore considered as practical tool to detect the effects of different parameters, measurement conditions, processing and probe placement on DPOAE levels. It has also great potential for development of new protocols, software, and hardware that can be used in new application areas.

\section{Acknowledgement}

Mirjana Adnadjevic is a recipient of the Special Research Fund (BOF) scholarship; the support of this organization is gratefully acknowledged. 
Annelies Bockstael is a postdoctoral fellow of the Research FoundationFlanders (FWO); the support of this organization is gratefully acknowledged.

Vincent Nadon is a recipient of the Institut Robert-Sauvé en santé et sécurité au travail (IRSST) Doctoral scholarship; the support of this organization is gratefully acknowledged.

\section{References}

[1] R. Probst, B. Lonsbury-Martinr, and G. Martin, "A review of otoacoustic emissions", J. Acoust. Soc. Am. 89, 2027-2067 (1991).

[2] M. Whitehead, B. Stagner, B. Lonsbury-Martin, G. Martin, "Measurement of otoacoustic emissions for hearing assessment", IEEE Eng. Med. Biol. Mag. 13, 210-226 (1994).

[3] M. S. Robinette and T. J. Glattke, "Otoacoustic emissions: clinical applications" (Thieme, New York, 2007), Chap. 1, pp. 1-48.

[4] O. V. Akinpelu, E. Peleva, W. R. J. Funnell, S. J. Daniel, "Otoacoustic emissions in newborn hearing screening: A systematic review of the effects of different protocols on test outcomes", Int. J. Pediatr. Otorhi. 78, 711-717 (2014).

[5] J. W. Hall, III, "Handbook of Otoacoustic Emissions" (Cengage Learning, 2000), Chap. 4, pp. 95-162.

[6] IEC 60645-6 Ed. 1.0 b:2009: "Electroacoustics - Audiometric equipment

- Part 6: Instruments for the measurement of otoacoustic emissions" (International Electrotechnical Commission, 2009). 
[7] V. Nadon, A. Bockstael, D. Botteldooren, J.-M. Lina, and J. Voix, "Individual monitoring of hearing status: Development and validation of advanced techniques to measure otoacoustic emissions in suboptimal test conditions", Appl. Acoust. 89, 78-87 (2015).

[8] L. Marshall, J. Lapsley Miller, and L. Heller, "Distortion-product otoacoustic emissions as a screening tool for noise-induced hearing loss", Noise Health 3, 43-60 (2001).

[9] Y. Liu and S. Neely, "Distortion product emissions from a cochlear model with nonlinear mechanoelectrical transduction in outer hair cells", J. Acoust. Soc. Am. 127, 2420-2432 (2010).

[10] S. T. Neely, T. A. Johnson, J. Kopun, D. M. Dierking, M. P. Gorga, "Distortion-product otoacoustic emission input/output characteristics in normal-hearing and hearing-impaired human ears", J. Acoust. Soc. Am. 126, 728-738 (2009).

[11] M. J. Rapson, J. C. Tapson, D. Karpul, "Unification and extension of monolithic state space and iterative cochlear models", J. Acoust. Soc. Am. 131, 3935-3952 (2012).

[12] J. A. How, S. J. Elliott, B. Lineton, "The influence on predicted harmonic and distortion product generation of the position of the nonlinearity within cochlear micromechanical models", J. Acoust. Soc. Am. 127, 652-655 (2010).

[13] F. Harris, B. Lonsbury-Martin, B. Stagner, A. Coats, G. Martin, "Acoustic distortion products in humans: Systematic changes in am- 
plitude as a function of $f_{2} / f_{1}$ ratio", J. Acoust. Soc. Am. 85, 220-229 (1989).

[14] A. Moulin, "Influence of primary frequencies ratio on distortion product otoacoustic emissions amplitude. II. Interrelations between multicomponent DPOAEs, tone-burst-evoked OAEs, and spontaneous OAEs", J. Acoust. Soc. Am. 107, 1471-1486 (2000).

[15] P. Dorn, D. Konrad-Martin, S. Neely, D. Keefe, E. Cyr, M. Gorga, "Distortion product otoacoustic emission input/output functions in normalhearing and hearing-impaired human ears", J. Acoust. Soc. Am. 110, 3119-3131 (2001).

[16] P. Avan, P. Bonfils, L. Gilain, T. Mom, "Physiopathological significance of distortion-product otoacoustic emissions at 2f1-f2 produced by highversus low-level stimuli", J. Acoust. Soc. Am. 113, 430-441 (2003).

[17] M. Adnadevic, A. Bockstael, D. Botteldooren, "Generic acoustic test fixture for oto-acoustic emissions: principles and design", Forum Acusticum, Krakow, Poland, (2014).

[18] H. Keppler, I. Dhooge, P. Corthals, L. Maes, W. D'haenens, A. Bockstael, B. Philips, F. Swinnen, B. Vinck, "The effects of aging on evoked otoacoustic emissions and efferent suppression of transient evoked otoacoustic emissions", Clin. Neurophysiol. 121, 359-365 (2010).

[19] A. Bockstael, H. Keppler, D. Botteldooren, "Improved hearing conservation in industry: more efficient implementation of distortion product 
otoacoustic emissions for accurate hearing status monitoring", Proceedings of Meetings on Acoustics,19, Montréal, QC, Canada, (2013).

[20] S. Puria, "Measurements of human middle ear forward and reverse acoustics: implications for otoacoustic emissions", J. Acoust. Soc. Am. 113, 2773-2789 (2003).

[21] M. Gorga, S. Neely, D. Dierking, J. Kopun, K. Jolkowski, K. Groenenboom, H. Tan,B. Stiegemann, "Low-frequency and high-frequency distortion product otoacoustic emission suppression in humans .", J. Acoust. Soc. Am. 123, 2172-2190 (2008).

[22] B. R. Glasberg, B. C. J. Moore, "Derivation of auditory filter shapes from notched-noise data", Hear. Res. 47, 103-138 (1990).

[23] C. A. Shera, J. J. Jr. Guinan, A. J. Oxenham, "Revised estimates of human cochlear tuning from otoacoustic and behavioral measurements", Proc. Natal. Acad. Sci. 99, 3318-3323 (2002).

[24] V. Chan, E. Wong, B. Mcpherson, "Occupational hearing loss: screening with distortion-product otoacoustic emissions", Int. J. Audiol. 43, 323329 (2004).

[25] H. Keppler, I. Dhooge, L. Maes,W. D’haenens, A. Bockstael,B. Philips, F. Swinnen, B. Vinck, "Transient-evoked and distortion product otoacoustic emissions: A short-term test-retest reliability study", Int. J. Audiol. 49, 99-109 (2010).

[26] A. Bockstael, B. de Greve, T. Van Renterghem, D. Botteldooren, W. D'haenens, H. Keppler, L. Maes, B. Philips, B. Vinck, "Verifying 
the attenuation of earplugs in situ: method validation using artificial head and numerical simulations", J. Acoust. Soc. Am. 124, 973-981 (2008).

[27] Y. Salimpour, M. Abolhassani, A. Ahmadian, K. Barin, "Multiresolution analysis of transient evoked otoacoustic emission", Conf. Proc. IEEE Eng. Med. Biol. Soc., 2773-2776 (2005).

[28] W. Ma and Y. Zhang, "Estimation of distortion product otoacoustic emissions", IEEE Trans. Biomed. Eng. 46, 1261-1264 (2002). 